\title{
(3) Gitumente

\section{Embedded ECG Based Real Time Monitoring and Control of Driver Drowsiness Condition}

\author{
M. Sangeetha ${ }^{1}$, S. Kalpanadevi ${ }^{2}$, M. Rajendiran ${ }^{2}$, G. Malathi \\ ${ }^{1}$ Embedded System Technologies, Knowledge Institute of Technology, Salem, India \\ ${ }^{2}$ Electrical and Electronics Engineering, Knowledge Institute of Technology, Salem, India
}

Email address:

msangeethaece82@gmail.com (M. Sangeetha), skeee@kiot.com (S. Kalpanadevi)

\section{To cite this article:}

M. Sangeetha, S. Kalpanadevi, M. Rajendiran, G. Malathi. Embedded ECG Based Real Time Monitoring and Control of Driver Drowsiness Condition. International Journal of Science, Technology and Society. Vol. 3, No. 4, 2015, pp. 146-150. doi: 10.11648/j.ijsts.20150304.17

\begin{abstract}
In recent years preventing accidents under drowsiness state has become a major focus for active safety driving. To reduce the accidents rate, it is needed to provide an efficient safety measure. Literature says that, the drowsiness condition of driver is best monitored by using an eye blink sensor (or) fabric electrode (or) ECG Sensor. But by monitoring the drowsiness condition alone, the accidents cannot be avoided, unless vehicle speed is controlled. To overcome this problem, the proposed system is implemented with a handheld hardware model designed with ARM and an embedded electrocardiogram electrode which measures the heart rate of the driver. The electrode is fixed in the right thumb and left index finger of the driver to monitor the drowsiness condition. If the system identifies that the driver is in drowsy state, it follows the sequence of operation like alters the driver by a buzzer, sends a warning signal to the control room, monitor and control the speed of the vehicle by cutting the fuel supply to the engine. In addition to that, the LED fixed at the backside of vehicle alerts the rear vehicles to reduce its speed. The proposed idea verified using Lab VIEW software is also discussed.
\end{abstract}

Keywords: ARM, ECG Electrode, DC Motor, GSM

\section{Introduction}

Now-a-days, the increasing number of transportation accidents has become a serious problem in society. Literature says that, the driver drowsiness state is one of the important factors for causing accidents. Hence drowsiness detection system finds an potential application in intelligent vehicle systems. Previous approach to drowsiness detection primarily makes pre-assumptions about the relevant behavior, focusing on blink rate, eye closure, and yawning from reference [1] and [2]. This paper, proposes a real time ECG based system for drowsiness detection.

All the above said works are recommended for monitoring the drowsiness state and alerting the driver by buzzer. Simply by monitoring the drowsiness condition of the driver alone is off no use, unless some control strategies is used for controlling the vehicle speed, due to which accidents can be avoided. To execute this, a GSM based embedded control system is proposed to monitor and control the parameters selected using its built-in input and output peripherals. The use of GSM is to receive the information transmitted and processing it further as required to perform several operations.
Depending on the nature of the information send the sequence of operations are to be performed like, the transmitted information is stored and polled from the receiver control station and then the required control signal is generated. This signal is send to the intermediate hardware that is designed using ARM and the signal is processed accordingly to perform the required task.

\section{Literature Survey}

\subsection{Sang-Joong Jung et al}

This paper describes about the driver health condition monitoring system and it alerts the driver under drowsiness condition. They proposed a new embedded electrocardiogram (ECG) sensor with electrically conductive fabric electrodes which can be fixed on the steering wheel of a car to monitor the driver's health condition.

The ECG signal were measured at a sampling rate of 100 $\mathrm{Hz}$ from the driver's palm as they stay on the pair of conductive electrodes located on the steering wheel. Practical 
tests were conducted using an embedded ECG sensor with a wireless sensor node, and the performances were assessed under non-stop two hours driving test. The driver's health condition such as normal, fatigued and drowsy states were analyzed by evaluating the heart rate variability in the time and frequency domains. But the vehicle speed is not controlled to avoid accidents caused by drowsiness state. [1]

\subsection{Jayanthi et al}

This system proposes an integrated method to detect driver's low alertness by considering both the performance of vehicle steering and the inattention. They proposed a new image processing algorithm designed to efficiently estimate driver's gaze direction, which is the facial inattention indicator adopted in this work.

Driver performance is measured according to the manipulation behavior of steering wheel. These two different aspects are generally complementary and thus can be integrated to obtain accurate detection. It can be implemented as a road device to the use of non-intrusive techniques. However, it is difficult to estimate driver's distraction or drowsiness accurately from the observation of those aspects.[2]

\subsection{Swapnil et al}

This paper proposed driver's fatigue approach for real-time detection of driver towards the driver's fatigue .The system consists of a sensor directly pointed towards the driver's face. The input to the stream is a continuous stream of signals from the sensors. It monitors the driver eyes to detect microsleeps, and monitors the driver jaw movement and it also detects the driver pulse from finger using LED and LDR assembling for detecting the driver illness. Driver fatigue levels based on the response signals were obtained which alerts the driver. It is expensive to be commercialized and needs complex noise processing. [7]

\subsection{Daniel Haupt et al}

This paper describes about reliable system for driver's drowsiness recognition. Unfortunately, majority of research work are carried out with data acquired in laboratory under ideal or simulated conditions. Therefore it is difficult to implement their results to real car and prove its reliability and accuracy. In this paper data taken for analysis is acquired from real traffic and therefore it overcomes all disadvantages partially modeled in laboratory. Here data acquisition has been chosen as an in-direct measurement from car CAN bus. All data are preprocessed according to assumptions about driver's behavior and transformed to frequency domain by means of orthogonal transform (STFT, CWT and DWT). Subsequently, data is analyzed by data mining methods including features extraction and filter feature selection. The performance of the feature is measured by the area under the receiver operating characteristics. Especially in Noninvasive systems the accuracy of driver's drowsiness detection is not sufficient. [4]

\section{Materials and Methods}

The overall block diagram of the proposed system for transmitter section and receiver section is shown in fig 1 and 2 respectively. This system comprises of ECG electrode, GSM, DC motor, alarm and control circuitry. It has transmitter and receiver section. In the transmitter section, an embedded electrocardiogram electrode is fixed on the right thumb and left index fingers of the driver to detect the ECG signal. An electrocardiogram electrode is a recording of the electrical activity of the heart over time produced by an electrocardiograph. Since the measured ECG signal is off low level, the amplifier amplifies the signal and sends to ARM 8 processor.

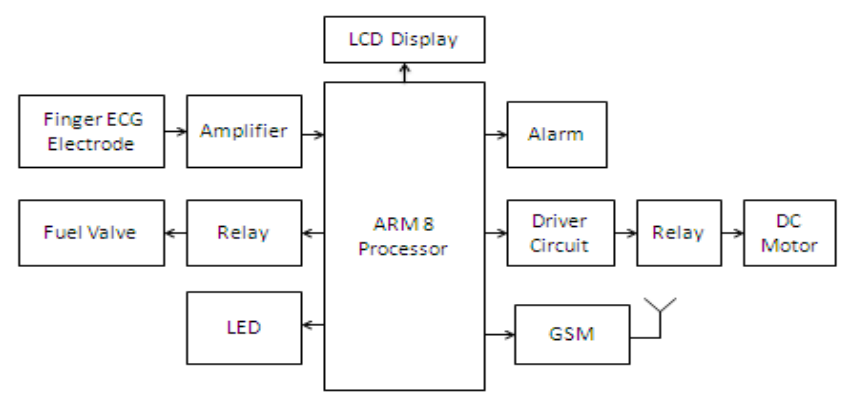

Fig. 1. Transmitter Section.

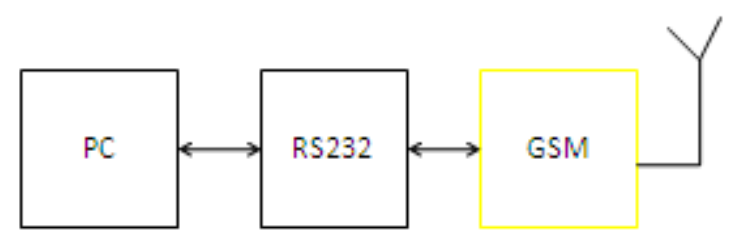

Fig. 2. Receiver Section.

\subsection{Block Diagram Description}

The ARM 8 processor supports a wide range of performance and it has the ability to scale in speed from $600 \mathrm{MHz}$ to greater than $1 \mathrm{GHz}$. It receives the amplified signal and process it accordingly. Here global system for mobile communication is used which has high performance, low consumption and long distance communication. By using GSM, these signals are transmitted to control room continuously. Based on the comparison between the received signal and the stored database in the control station, the signal will be send back to the vehicle through GSM to carry out further action. If the received signal indicates the driver is in drowsiness state, then the processor will activate the driver circuit and it alerts the driver by producing an alarm.

The drowsiness prediction system uses DC motor instead of vehicle engine for to control the speed of the vehicle. The DC motor is connected to the processor using the relay control. When any abnormalities in the sensed values are detected by the processor, the relay is open and the motor stops running.

The LCD monitors the condition of the driver and LED fixed at the back side of the vehicle alert the following vehicles to reduce it speed. At the same time the relay will 
pass the control signal to the fuel valve to cut-off the fuel given to the engine to stop it operation temporarily.

\subsection{Algorithm}

Step 1: Start the program.

Step 2: Initialization the system. Check whether the driver is in normal state or in abnormal state.
Step 3: If the driver is in drowsiness state the alarm will be intimate otherwise again go to step 2.

Step 4: Check the heart rate, if it in drowsiness state it displays condition of the driver.

Step 5: Stop the Program.

\subsection{Flow Chart}
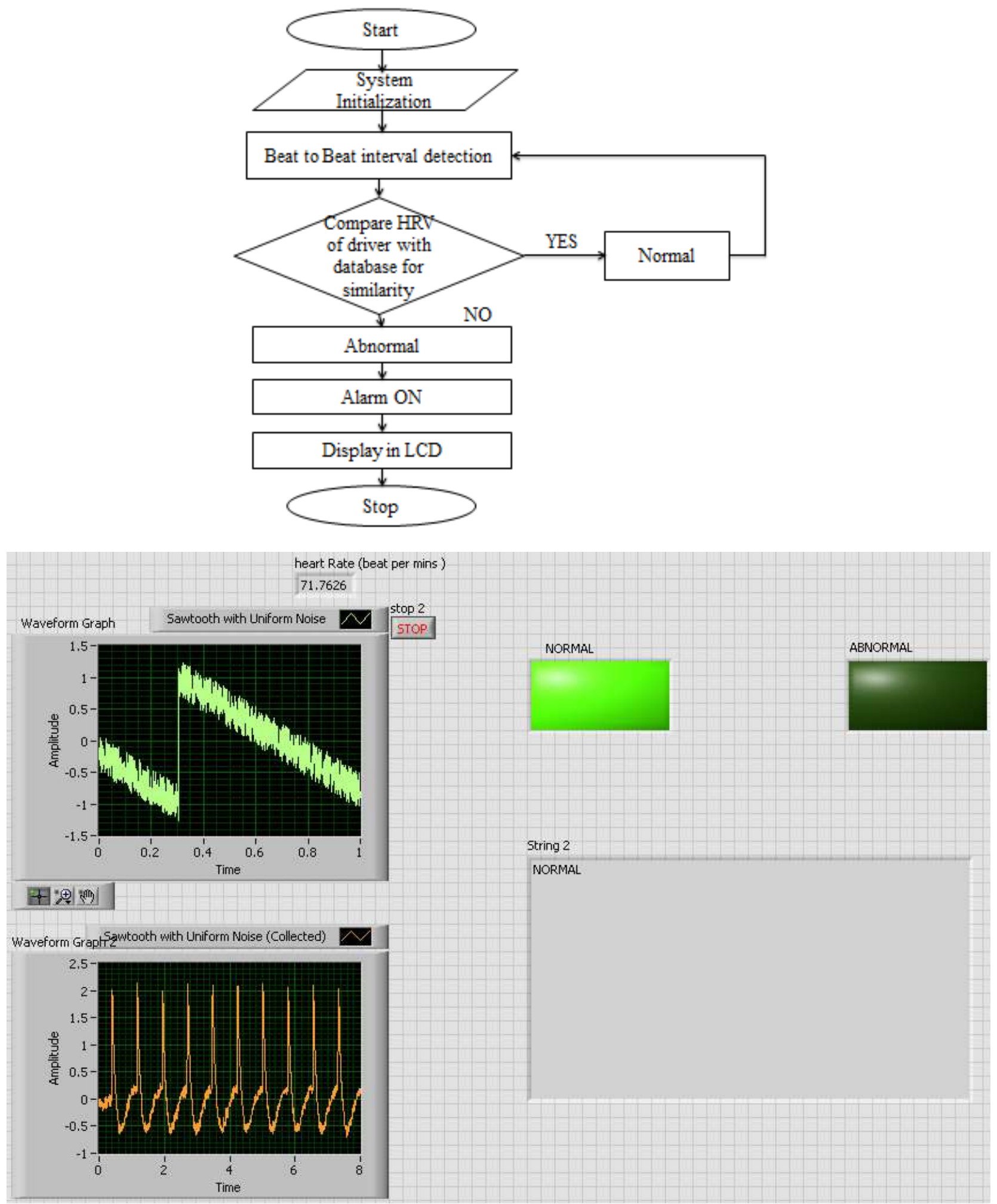

Fig. 3. Normal State. 


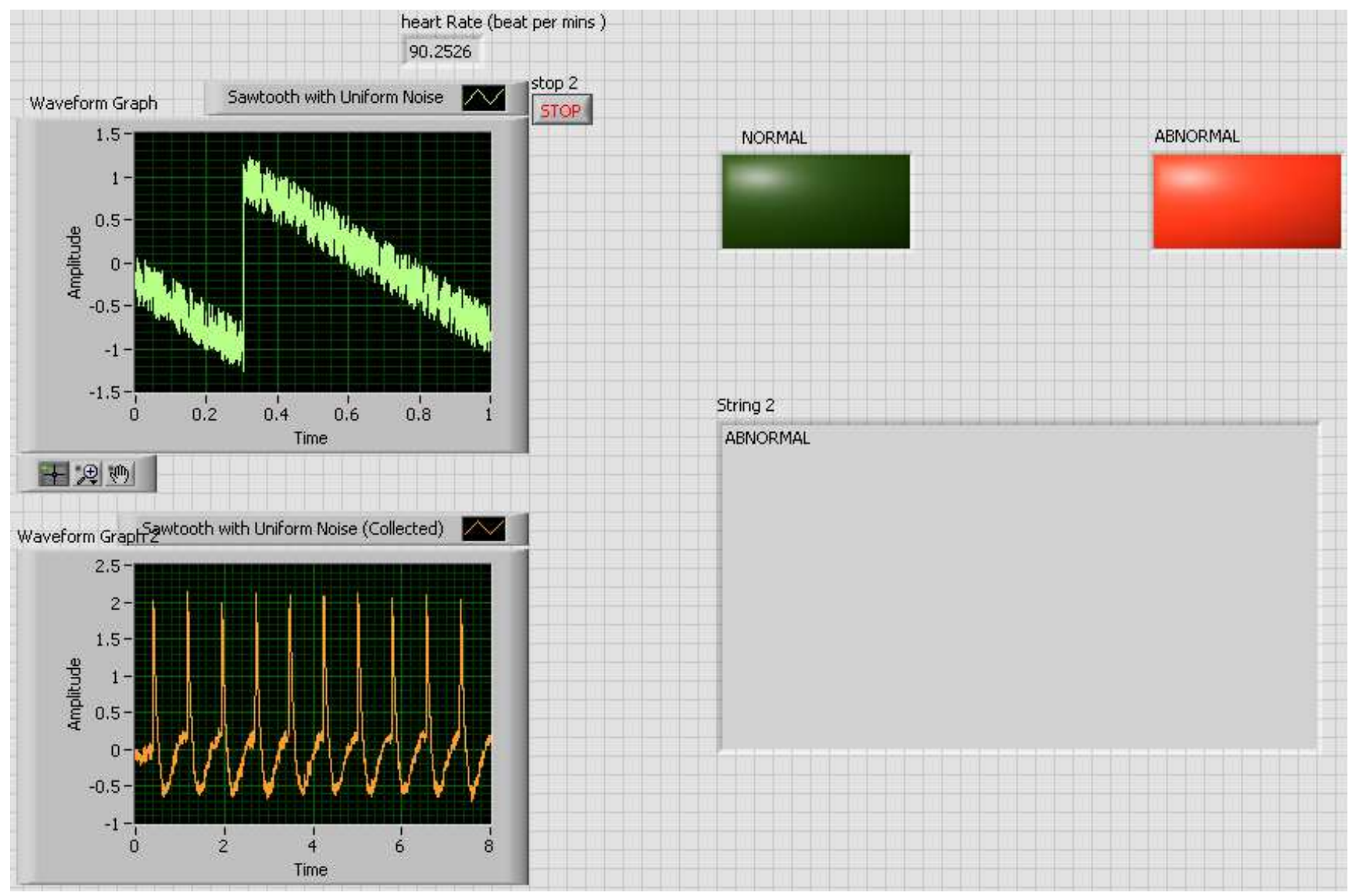

Fig. 4. Abnormal State.

\section{Results and Discussion}

\section{Software Results}

The proposed idea is simulated using Lab VIEW software. The ECG signal used in this simulation is the normal heartbeat signal of the driver which is stored in database. By analyzing the actual HRV signal with database signal. It indicates whether the driver is in normal or abnormal condition and it is graphically represented using Lab VIEW. If the comparison result indicates the abnormal condition, it is displayed using the graphical method. If the heart rate is 72 beats per second, it indicates the normal condition. Fig. 3 and 4 which is shown below displays the ECG signal of the driver under normal and abnormal condition.

\section{Conclusion}

The idea proposed gives a novel embedded drowsiness detection system to monitor and control the human cognitive state and to provide biofeedback to stop the vehicle when drowsiness state is detected. This system is suitable for all automobile applications with an intention to save the human life by avoiding accidents rates. The proposed idea was successfully verified through simulation using Lab VIEW.

\section{Acknowledgment}

The authors wish to thank the management of Knowledge Institute of Technology and Head of the department for providing the facilities to carry out this work.

\section{References}

[1] Sang-Joong, Heung-Sub Shin, Wan-Young Chung "Driver fatigue and drowsiness monitoring system with embedded electrocardiogram sensor on steering wheel" IET intell. Transp. Syst, 2014, Vol.8.

[2] D. Jayanthi, M. Bommy "Vision based real time driver fatigue detection system for efficient vehicle control" (IJEAT, Vol-2, 2012).

[3] DeRosario. H, Solaz. J. S., odríguez: "Controlled inducement and measurement of drowsiness in adriving simulator" (IETIntell. Transp) 2010, 4, pp. 280-288).

[4] Daniel Haupt, petrHonzix, Peter Raso"Steering wheel motion analysis for detection of the driver's drowsiness".

[5] Jia-Xiu Liu, Ming-KuanKo"Detection of driver's low vigilance by analyzing driving behaviour from multi-aspects" (National Conference, Vol-14, 2012).

[6] Chin-Teng Lin, wen-Hung Chao, Yu-Jie Chen"EEG-based drowsiness estimation for safety driving using independent component analysis" (IEEE Transactions, Vol-52, 2005). 
[7] Swapnil, Deepak, Kapil "Driver fatigue detection using sensornetwork" (IJEST, 2011).

[8] Federico Baronti, Roberto Roncella, Roberto Saletti“ Distribetded sensor for steering wheel grip force measurement in driver fatigue detection" (EDAA, 2009).
[9] Betsy Thomas, Ashutosh Gupta "Wireless sensor embedded steering wheel for real time monitoring of driver fatigue detection"(International Conference, 2012). 